\title{
Gemcitabine Plus Nedaplatin as Salvage Therapy is a Favorable Option for Patients with Progressive Metastatic Urothelial Carcinoma After Two Lines of Chemotherapy
}

\author{
Kazumasa Matsumoto*, Kohei Mochizuki, Takahiro Hirayama, Masaomi Ikeda, \\ Morihiro Nishi, Ken-ichi Tabata, Miyoko Okazaki, Tetsuo Fujita, Yoshinori \\ Taoka, Masatsugu Iwamura
}

\begin{abstract}
This study was conducted to evaluate the effectiveness of a combination of gemcitabine and nedaplatin therapy among patients with metastatic urothelial carcinoma previously treated with two lines of chemotherapy. Between February 2009 and August 2013, 30 patients were treated with gemcitabine and paclitaxel as a second-line chemotherapy. All had received a first-line chemotherapy consisting of methotrexate, vinblastine, doxorubicin and cisplatin. Ten patients who had measurable histologically proven advanced or metastatic urothelial carcinoma of the urinary bladder and upper urinary tract received gemcitabine $1,000 \mathrm{mg} / \mathrm{m}^{2}$ on days 1,8 and 15 and nedaplatin $70 \mathrm{mg} / \mathrm{m}^{2}$ on day 2 as a third-line chemotherapy. Tumors were assessed by imaging every two cycles. The median number of treatment cycles was 3.5. One patient had partial response and three had stable disease. The disease-control rate was $40 \%$, the median overall survival was 8.8 months and the median progression-free survival was 5.0 months. The median overall survival times for the first-line and second-line therapies were 29.1 and 13.9 months, respectively. Among disease-controlled patients $(n=4)$, median overall survival was 14.2 months. Myelosuppression was the most common toxicity. There were no therapy-related deaths. Gemcitabine and nedaplatin chemotherapy is a favorable third-line chemotherapeutic option for patients with metastatic urothelial carcinoma. Given the safety and benefit profile seen in this study, further prospective trials are warranted given the implications of our results with regard to strategic chemotherapy for patients with advanced or metastatic urothelial carcinoma.
\end{abstract}

Keywords: Bladder - chemotherapy - upper urinary tract - urothelial carcinoma

Asian Pac J Cancer Prev, 16 (6), 2483-2487

\section{Introduction}

Urothelial carcinoma (UC) is the most common cancer of the urinary tract. In patients with muscle invasive lesions, recurrence or progression is common after radical surgery and perioperative chemotherapy. Metastatic UC is relatively sensitive to chemotherapeutic agents and platinum-based chemotherapy represents the standard first-line therapy (Hussain et al., 2003). However, the median overall survival (OS) for patients with metastatic UC of the urinary bladder is only approximately 15 months (von der Maase et al., 2005). This poor survival is due mainly to the lack of an effective salvage treatment strategy.

There is no established treatment for patients with progressive disease after first-line platinum-based chemotherapy. Recently, treatment with vinflunine plus best supportive care (BSC) was demonstrated to significantly improve OS in eligible patients compared to patients who received BSC alone in a phase III trial and this treatment has been approved in Europe as a secondline therapy for advanced UC (Bellmunt et al., 2009). The median OS for patients treated with vinflunine plus BSC was 6.9 months. Since gemcitabine and paclitaxel (GP) was adopted in 2005 as a second-line chemotherapy after methotrexate, vinblastine, doxorubicin and cisplatin (MVAC) at our institution, this regimen has proved to be well-tolerated and to have the potential to prolong survival among patients with UC (Matsumoto et al., 2007; Ikeda et al., 2011). However, the differential effect of the number of prior lines of treatment and the setting of prior therapy in patients receiving salvage chemotherapy for advanced $\mathrm{UC}$ is unclear.

Nedaplatin is a second-generation platinum derivative that shows antitumor activity equivalent to that cisplatin, but its side effects, such as nausea, nephrotoxicity and 
neurotoxicity, are less severe (Kameyama et al., 1990; Li et al., 2014). Gemcitabine, which is a nucleoside analog related to cytarabine, is a pyrimidine antimetabolite (Jamshed et al., 2014) and has been shown to be one of the most effective cytotoxic agents against UC (Matsumoto et al., 2007; Ikeda et al., 2011). Gemcitabine and nedaplatin (GN) therapy shows greater activity against tumor models than the combination of gemcitabine with cisplatin or carboplatin (Matsumoto et al., 2001).

Some patients whose disease progresses after a secondline chemotherapy are willing to receive additional cancercontrol treatment in addition to BSC. However, to our knowledge, there have been only two previous studies of the role of chemotherapy after second-line chemotherapy (Soga et al., 2010; Rozzi et al., 2013). In this study, our aim was to determine whether GN that is reacted with antitumor mechanism is an effective treatment for patients with metastatic UC who have previously been treated with GP followed by MVAC. Specifically, the primary objective of the study was to assess OS among patients treated with GN as a third-line therapy. Secondary objectives were to evaluate tumor response, duration of response, time to treatment failure and toxicity.

\section{Materials and Methods}

\section{Patients}

Patients had measurable or assessable cancerous lesions which were histologically proven to be locally advanced or metastatic UC. All patients had received surgical treatment or biopsy of the primary lesions and chemotherapy consisting of GP followed by MVAC. Patients were required to have a eastern cooperative oncology group performance status (PS) of 2 or lower per world health organization criteria; adequate bone marrow reserve (white blood cell (WBC) count $>3,500 /$ $\mu \mathrm{L}$, platelet count $>100,000 / \mu \mathrm{L}$, hemoglobin $>10 \mathrm{~g} / \mathrm{dL}$ ), adequate hepatic function (serum bilirubin $\leq 1.5 \mathrm{mg}$ / $\mathrm{dL}$ ), adequate renal function (serum creatinine $1.5 \mathrm{mg}$ / $\mathrm{dL}$ or measured creatinine clearance of $\geq 60 \mathrm{~mL} / \mathrm{min}$ ) and an estimated life expectancy of at least 12 weeks. Patients with central nervous system metastases, second primary malignant lesions, or clinically significant pleural effusions or ascites, nonmalignant systemic disease that precluded them from receiving therapy, including active infection, any clinically significant cardiac arrhythmia or congestive heart failure, were not eligible.

All patients gave written informed consent before entering the study, which was approved by the institutional chemotherapy review board at Kitasato university hospital.

\section{Treatment protocol}

The GN protocol was similar to the gemcitabine and cisplatin (GC) protocol (von der Maase et al., 2005). All patients received gemcitabine $1,000 \mathrm{mg} / \mathrm{m}^{2}$ on days 1,8 and 15 and nedaplatin $70 \mathrm{mg} / \mathrm{m}^{2}$ on day 2 and the treatment cycle was repeated every 4 weeks. In each cycle, the full dose of nedaplatin was given if the WBC count was $>3000 / \mu \mathrm{L}$ and the platelet count was $>100,000 / \mu \mathrm{L}$. If the counts were lower than these levels, treatment protocol was delayed for 1 week. On days 8 and 15 of each cycle, full-dose gemcitabine was given if the patients had a WBC count of $>3,000 / \mu \mathrm{L}$ and a platelet count of $>75,000 / \mu \mathrm{L}$. All patients were allowed to receive antiemetics such as dexamethasone and granisetron hydrochloride and posttherapy hydration with $1,000 \mathrm{~mL}$ of normal saline.

Supportive care could include blood transfusion and analgesics. Prophylactic use of granulocyte colonystimulating factor was not used. If after the first cycle, the toxicity levels exceeded those specified in the eligibility criteria, the subsequent cycles were delayed until the levels returned to the specified levels. If an adverse event classified as grade 2 or higher occurred, the doses of both drugs were decreased, by $25-50 \%$, for the subsequent cycle. Further local therapy, including radiation therapy, was allowed for patients with locally advanced disease after their responses to the GN protocol were evaluated.

\section{Treatment evaluation}

During treatment, blood counts and serum chemistry were assessed weekly and creatinine clearance was calculated before chemotherapy. Tumors were assessed by computerized tomography or magnetic resonance imaging every two cycles.

On the basis of patient medical records, OS was measured until death and time to failure was measured until discontinuation of treatment, death, or disease progression. Patients were assigned a response category according to the Response Evaluation Criteria in Solid Tumors guideline (ver. 1.1). Complete response (CR) was defined as the disappearance of all target lesions and reduction of any pathological lymph nodes (whether target or nontarget) to $<10 \mathrm{~mm}$ in the short axis. Partial response (PR) was defined as a decrease in the sum of diameters of the target lesions by at least $30 \%$. Progressive disease (PD) was defined as an increase in the sum of the diameters of the target lesions by at least $20 \%$; in addition to the relative increase of $20 \%$, the sum of the diameters also had to demonstrate an absolute increase of at least $5 \mathrm{~mm}$. The appearance of one or more new lesions was also defined as PD. Stable disease (SD) was defined as neither sufficient shrinkage to qualify for PR nor sufficient increase to qualify for PD.

Adverse events were monitored according to the National Cancer Institute Common Terminology Criteria for Adverse Events (ver. 3.0).

\section{Statistical analysis}

OS rate and response duration were calculated from the first day of GN therapy until the date of progression or death. OS rate from previous chemotherapy was calculated from the first day of MVAC or GP therapy until the date of death. Survival curves were analyzed by means of the Kaplan-Meier method using the log-rank test. All analyses were performed with StatView (ver. 5.0, SAS Institute, Cary, NC, USA) and $\mathrm{p}<0.05$ was considered statistically significant.

\section{Results}

Patients' characteristics

Between february 2009 and august 2013, 30 patients 
were treated with the GP as a second-line chemotherapy. Before GP, all the patients had received one previous chemotherapy that consisted of an MVAC. Twelve patients of the 30 patients received GN as a third-line chemotherapy. Of these, two were excluded because they showed progressive diseases during only one cycle of GN. Of the remaining ten patients (Table 1), eight were men and two were women; the median age was 67 years (range 53-82 years). Five patients had bladder UC, three had upper urinary tract (UUT) UC and two had both lesions. After the GP, four patients had multiple lymph node metastases, one had lung metastases and five had two or more visceral metastases.

\section{Treatment received}

Ten eligible patients received at least two cycles of GN and were evaluated for response. The median number of cycles was 3.5 (range 2-4). The median number of MVAC before GP was four (range 3-4) and the median number of GP before GN was three (range 2-7). Over the course of the study, 31 cycles of GN were administered. The median doses of gemcitabine and nedaplatin were 750 $\mathrm{mg} / \mathrm{m}^{2}$ (range $490-1,000 \mathrm{mg} / \mathrm{m}^{2}$ ) and $52.5 \mathrm{mg} / \mathrm{m}^{2}$ (range $35-70 \mathrm{mg} / \mathrm{m}^{2}$ ), respectively. Dose reduction was required in all patients. The percentage of the planned treatment of day 8 and 15 were $70 \%$ and $53 \%$, respectively. The most common reason for omitting a planned treatment was myelosuppression.

\section{Treatment efficacy}

The objective tumor responses to the three chemotherapies are shown in Table 2. One patient showed $\mathrm{PR}$ to GN, none showed $\mathrm{CR}$ and the overall response rate was $10 \%$. The disease-control rate (PR and SD) for GN was $40 \%$. The median OS was 8.8 months (range 2.8-19.2 months). The survival rate in the first year of follow up was $30 \%$. The median progression-free survival (PFS) was 5.0 months (range 1.6-14.3 months). The median OS times for MVAC and GP were 29.1 months (range 11.055.7 months) and 13.9 months (range 6.1-33.1 months), respectively. Three patients showed brain metastases after failure of the GN therapy.

The ten patients were subdivided into two groups: a controlled-disease group consisting of patients who showed PR or $\mathrm{SD}(\mathrm{n}=4)$ and an uncontrolled-disease group consisting of patients who showed PD $(n=6)$. Median OS times in the controlled-disease and uncontrolled-disease groups after GN were 14.2 and 6.2 months, respectively (Figure 1), but the difference between the groups was not statistically significant $(\mathrm{p}=0.08)$.

\section{Adverse events}

Hematological and nonhematological adverse events experienced by the ten patients are listed in Table 3 . Myelosuppression was the most common adverse event. Grade 3 neutropenia occurred in four patients and grade 4 occurred in one patient. The patients were given granulocyte colony-stimulating factor and responded to it well. Febrile neutropenia was observed in two patients. There were no severe infections. Grade 3 thrombocytopenia occurred in four patients and one patient experienced grade 4 thrombocytopenia and required platelet transfusions, but there were no episodes of bleeding. There were no severe nonhematological adverse events and there were no treatment-related deaths.

\section{Discussion}

One benefit of allowing patients who have received prior chemotherapy and multiple prior lines of therapy is acceleration of trial accrual and drug development, which are generally slow, owing to poor survival and comorbidities in patients with advanced or metastatic UC. In this study, we demonstrated that median OS and PFS after GN as a third-line therapy were 8.8 and 5.0 months, respectively. Among patients with controlled disease, OS was 14.2 months. Median OS times for the MVAC and GP therapies were 29.1 and 13.9 months, respectively. Furthermore, third-line GN therapy was safe and well tolerated. These data indicate that an intensive chemotherapy strategy for patients with advanced or metastatic UC has the potential to prolong survival, with a high proportion of patients maintaining a quality of life.

Pond et al. reported on the impact of the number of prior lines of chemotherapy on prognosis among patients with advanced UC (Pond et al., 2014). In the data set used by these investigators, the numbers of prior lines of chemotherapy, including perioperative chemotherapy, were as follows: one in $78.6 \%$ of the patients, two in $15.6 \%$, three in $4.1 \%$, four in $1.4 \%$ and five in $0.3 \%$. That is, most of the patients had received two or fewer prior lines; only $5.8 \%$ of patients had received more than 2 and only $1.7 \%$ had received more than three. Receiving several lines of prior treatment might increase the risk of toxicities and might reduce survival, owing to the large number of chemotherapeutic agents. However, Pond et al. showed that the number of prior lines did not have a significant impact on clinical efficacy outcomes, which suggests that toxicities probably did not increase to an extent sufficient to compromise survival. Because people are living longer which increases their risk of cancer and there are many new chemotherapeutic agents, patients have higher expectations with regard to the effectiveness of cancer treatments. In addition, patients with metastatic $\mathrm{UC}$ are willing to receive additional treatments beyond second-line chemotherapy, particularly in middle-aged

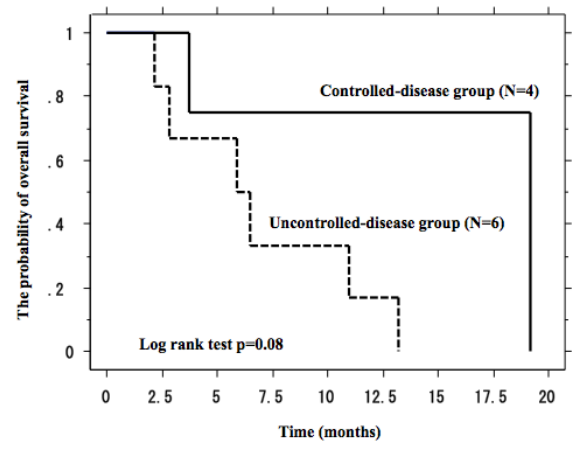

Figure 1. Overall Survival in Patients with ControlledDisease (Partial Response or Stable Disease, $n=4$ ) and Uncontrolled-Disease $(\mathbf{n}=\mathbf{6})$. The median overall survival times for the two groups after gemcitabine and nedaplatin chemotherapy were 14.2 and 6.2 months, respectively. 


\begin{tabular}{rccclc}
\hline Case & Gender & Age & PS & Primary sites & Metastatic sites \\
\hline 1 & M & 73 & 0 & Bladder & Multiple sites \\
2 & M & 67 & 1 & Bladder & Lymph nodes \\
3 & M & 79 & 0 & UUT & Lymph nodes \\
4 & F & 67 & 0 & UUT & Lung \\
5 & M & 67 & 1 & Bladder \& UUT & Multiple sites \\
6 & M & 74 & 1 & Bladder \& UUT & Multiple sites \\
7 & M & 65 & 0 & UUT & Multiple sites \\
8 & M & 53 & 1 & Bladder & Multiple sites \\
9 & F & 64 & 0 & Bladder & Lung \\
10 & M & 82 & 1 & Bladder & Lymph nodes \\
\hline
\end{tabular}

*PS: Eastern cooperative oncology group performance status; UUT: upper urinary tract.

Table 2. Numbers of Treatment Cycles and Responses to the Three Chemotherapies Among Ten Patients with Urothelial Carcinoma.

\begin{tabular}{|c|c|c|c|c|c|c|}
\hline \multirow[t]{2}{*}{ Case } & \multicolumn{2}{|c|}{ MVAC } & \multicolumn{2}{|l|}{ GP } & \multicolumn{2}{|l|}{ GN } \\
\hline & Response & No. & Response & No. & Response & No. \\
\hline 1 & $\mathrm{CR}$ & 4 & SD & 2 & PD & 2 \\
\hline 2 & PD & 4 & SD & 2 & PD & 3 \\
\hline 3 & CR & 4 & SD & 4 & SD & 4 \\
\hline 4 & PD & 4 & SD & 2 & PD & 2 \\
\hline 5 & CR & 3 & PD & 4 & PD & 4 \\
\hline 6 & PR & 4 & PD & 4 & PD & 2 \\
\hline 7 & PD & 3 & PD & 2 & PD & 2 \\
\hline 8 & $\mathrm{CR}$ & 4 & SD & 7 & SD & 4 \\
\hline 9 & PR & 4 & SD & 2 & SD & 4 \\
\hline 10 & PR & 4 & PR & 5 & PR & 4 \\
\hline \multicolumn{7}{|c|}{ Response rate (\%) } \\
\hline & 70 & & 10 & & 10 & \\
\hline \multicolumn{7}{|c|}{ Disease control rate $(\%)$} \\
\hline & 70 & & 70 & & 40 & \\
\hline
\end{tabular}

*MVAC: methotrexate, vinblastine, doxorubicin and cisplatin; GP:gemcitabine and paclitaxel; GN:gemcitabine and nedaplatin; No.:number; CR: complete response; PR:partial resonse; SD:stable diseas; PD:progressive disease.

Table 3. Adverse Events Among Patients Treated with Gemcitabine and Nedaplatin as A Third-Line Chemotherapy.

\begin{tabular}{lcccc}
\hline Adverse event & \multicolumn{4}{c}{ Number of patients } \\
\cline { 2 - 5 } & Grade 1 & Grade 2 & Grade 3 & Grade 4 \\
\hline Hematological & 2 & 3 & 4 & 1 \\
$\quad$ Neutropenia & 1 & 3 & 4 & 1 \\
Thrombocytopenia & 1 & 3 & 2 & 1 \\
Leukopenia & 0 & 0 & 2 & 0 \\
Febrile neutropenia & 0 & 3 & 2 & 0 \\
Anemia & 1 & 1 & 1 & 0 \\
Lymphopenia & 0 & & & \\
Nonhematological & & 4 & 0 & 0 \\
$\quad$ Anorexia & 6 & 3 & 0 & 0 \\
Fatigue & 7 & 1 & 0 & 0 \\
$\quad$ Nausea/Vomiting & 5 & 1 & 0 & 0 \\
Elevated AST/ALT & 1 & 0 & 0 & 0 \\
$\quad$ Elevated creatinine & 1 &
\end{tabular}

patients, who would otherwise have a long life expectancy.

There have been two previous reports on third-line chemotherapy for UC. In the first reported study, Soga et al. evaluated the efficacy and toxicity of gemcitabine monotherapy as a third-line chemotherapy in 13 patients with advanced UC (Soga et al., 2010), the first-line therapy was MVAC and the second-line therapy was a combination of paclitaxel and carboplatin. One patient (7.7\%) showed
PR and seven patients (53.8\%) had SD. Median OS and PFS times were 7.3 and 2 months, respectively. Approximately $30 \%$ of the patients who received gemcitabine developed grade 3-4 hematological toxicities. In the second study, Rozzi et al. evaluated pegylated liposomal doxorubicin as a third-line chemotherapy in 23 patients who had already been treated with paclitaxel combined with carboplatin or paclitaxel monotherapy and then with GC (Rozzi et al., 2013). These investigators demonstrated that pegylated liposomal doxorubicin had a safe toxicity profile; no grade 4 hematological or nonhematological events occurred. Although three patients experienced PR, the diseasecontrol rate was $43 \%$, median OS was 6.3 months and median PFS was 4.1 months. In our study, GN therapy as a third-line chemotherapy showed a disease-control rate of $40 \%$ and median OS and PFS times of 8.8 and 5.0 months, respectively. Although direct comparison between our results and the results of the two previous studies is difficult, owing to the differences between patient backgrounds and treatment strategies, we suggest that the intensive treatment strategy consisting of MVAC, GP and GN may be a beneficial option for patients with advanced or metastatic UC.

As expected, neutropenia and thrombocytopenia were the major hematological toxicities in our study. Grade 3 or 4 neutropenia occurred in five patients, but there were no treatment-related deaths or overwhelming infections. Although grade 3 or 4 thrombocytopenia occurred in five patients, only one patient required platelet transfusions and there were no hemorrhages. Because the patients had received many courses of chemotherapy prior to receiving GN, anorexia and fatigue were the most frequent nonhematological toxicities, but they were mild. No patient discontinued the treatment owing to toxic effects. The relatively mild toxicity profile of GN therapy even as a third-line chemotherapy makes this combination an attractive strategy for progressive UC.

Brain metastasis occurs in less than $1 \%$ of patients with UC (Mahmoud-Ahmed et al., 2002). In our study, three patients developed brain metastases. They received no aggressive treatment after GN chemotherapy. The development of brain metastases can be attributed to the fact that even during long-term systemic remission, cancer cells within the central nervous system are protected from chemotherapeutic agents by the blood-brain barrier (Anderson et al., 1992). No large-cohort data exist regarding the treatment of brain metastases from UC. One study of patients $(n=62)$ with metastatic brain lesions has 
been reported;(Fokas et al., 2010) in this study, patients with brain metastases were treated with radiotherapy with or without surgery and the survival rate was poor. In the majority of prospective and retrospective assessments of the efficacy of chemotherapy in metastatic UC, patients with brain metastases either were not reported or were excluded from the study design (Kaufman et al., 2000; von der Maase et al., 2005). Although our study did not provide any clues regarding the treatment of brain metastasis, we may need to pay attention and to detect it early leading to keeping patients' quality of life after the number of lines of chemotherapy.

This study had several limitations, including the small sample size. In addition, all the patients received MVAC as a first-line chemotherapy; currently, more patients with advanced UC undergo GC rather than MVAC as a first-line chemotherapy because GC and MVAC have equivalent anticancer activity but GC shows lower toxicity (von der Maase et al., 2005). Despite the lack of a discernible significant impact of the number of prior lines of therapy on clinical outcomes, careful selection of a chemotherapy protocol, starting from the time of the treatment-naïve condition, on the basis of the specific chemotherapeutic agents administered is nevertheless warranted. For example, in future evaluations of GP therapy, patients would probably be stratified according to prior GC administration, given the probability of cross resistance. Another limitation of our study was the fact that when the GN therapy was administered on a 4 -week schedule, $47 \%$ of the patients did not receive gemcitabine on day 15 because they experienced increased myelosuppression. Some recent trials have reported good activity and tolerability of 3-week schedules for GC administration (Abratt et al., 1998; Castellano et al., 1998). The salvage GN chemotherapy evaluated in this study would also be acceptable on a 3-week schedule. Despite these limitations, our results suggest that GN as a third-line therapy is effective, safe and well-tolerated. Further prospective trials are warranted to implication of our results with regard to strategic chemotherapy for patients with advanced or metastatic urothelial carcinoma (Matsumoto et al., 2007; Ikeda et al., 2011; Ikeda et al., 2014).

\section{References}

Abratt RP, Sandler A, Crino L, et al (1998). Combined cisplatin and gemcitabine for non-small cell lung cancer: influence of scheduling on toxicity and drug delivery. Semin Oncol, 25, 35-43.

Anderson RS, el-Mahdi AM, Kuban DA, Higgins EM (1992). Brain metastases from transitional cell carcinoma of urinary bladder. Urology, 39, 17-20.

Bellmunt J, Theodore C, Demkov T, et al (2009). Phase III trial of vinflunine plus best supportive care compared with best supportive care alone after a platinum-containing regimen in patients with advanced transitional cell carcinoma of the urothelial tract. J Clin Oncol, 27, 4454-61.

Castellano D, Lianes P, Paz-Ares L, et al (1998). A phase II study of a novel gemcitabine plus cisplatin regimen administered every three weeks for advanced non-small-cell lung cancer. Ann Oncol, 9, 457-9.
Fokas E, Henzel M, Engenhart-Cabillic R (2010). A comparison of radiotherapy with radiotherapy plus surgery for brain metastases from urinary bladder cancer: analysis of 62 patients. Strahlenther Onkol, 186, 565-71.

Hussain SA, James ND (2003). The systemic treatment of advanced and metastatic bladder cancer. Lancet Oncol, 4, 489-97.

Ikeda M, Matsumoto K, Nishi M, et al (2014). Comparison of radical cystectomy and chemoradiotherapy in patients with locally advanced bladder cancer. Asian Pac J Cancer Prev, 15, 6519-24.

Ikeda M, Matsumoto K, Tabata K, et al (2011). Combination of gemcitabine and paclitaxel is a favorable option for patients with advanced or metastatic urothelial carcinoma previously treated with cisplatin-based chemotherapy. Jpn J Clin Oncol, 41, 1214-20.

Jamshed A, Hussain R, Iqbal H (2014). Gemcitabine and cisplatin followed by chemo-radiation for advanced nasopharyngeal carcinoma. Asian Pac J Cancer Prev, 15, 899-904.

Kameyama Y, Okazaki N, Nakagawa M, et al (1990). Nephrotoxicity of a new platinum compound, 254-S, evaluated with rat kidney cortical slices. Toxicol Lett, 52, 15-24.

Kaufman D, Raghavan D, Carducci M, et al (2000). Phase II trial of gemcitabine plus cisplatin in patients with metastatic urothelial cancer. J Clin Oncol, 18, 1921-7.

Kaufman DW, Shapiro S (2000). Epidemiological assessment of drug-induced disease. Lancet, 356, 1339-43.

Li CH, Liu MY, Liu W, et al (2014). Randomized control study of nedaplatin or cisplatin concomitant with other chemotherapy in the treatment of advanced non-small cell lung cancer. Asian Pac J Cancer Prev, 15, 731-6.

Mahmoud-Ahmed AS, Suh JH, Kupelian PA, et al (2002). Brain metastases from bladder carcinoma: presentation, treatment and survival. J Urol, 167, 2419-22.

Matsumoto K, Irie A, Satoh T, et al (2007). Gemcitabine and paclitaxel chemotherapy as a second-line treatment for advanced or metastatic urothelial carcinoma. Int J Urol, 14, 1000-4.

Matsumoto M, Takeda Y, Maki H, et al (2001). Preclinical in vivo antitumor efficacy of nedaplatin with gemcitabine against human lung cancer. Jpn J Cancer Res, 92, 51-8.

Pond GR, Bellmunt J, Rosenberg JE, et al (2014). Impact of the number of prior lines of therapy and prior perioperative chemotherapy in patients receiving salvage therapy for advanced urothelial carcinoma: implications for trial design. Clin Genitourin Cancer, 13, 71-9

Rozzi A, Santini D, Salerno M, et al (2013). Pegylated liposomal doxorubicin as third-line chemotherapy in patients with metastatic transitional cell carcinoma of urothelial tract: results of a phase II study. Med Oncol, 30, 407.

Soga N, Kise H, Arima K, Sugimura Y (2010). Third-line gemcitabine monotherapy for platinum-resistant advanced urothelial cancer. Int J Clin Oncol, 15, 376-81.

von der Maase H, Sengelov L, Roberts JT, et al (2005). Longterm survival results of a randomized trial comparing gemcitabine plus cisplatin, with methotrexate, vinblastine, doxorubicin, plus cisplatin in patients with bladder cancer. J Clin Oncol, 23, 4602-8. 